\title{
An Adaptive Observer-based Switched Methodology for the Identification of a Perturbed Sinusoidal Signal: Theory and Experiments
}

\author{
B. Chen, G. Pin, W. M. Ng, C. K. Lee, S. Y. R. Hui, and T. Parisini
}

\begin{abstract}
This paper deals with a novel adaptive observerbased technique for estimating the amplitude, frequency and phase of a single sinusoidal signal from a measurement affected by structured and unstructured disturbances. The structured disturbances are modelled as a time-polynomial so as to represent bias and drift phenomena typically present in applications, whereas the unstructured disturbances are modelled as bounded noise signals. The proposed estimation technique exploits a specific adaptive observer scheme equipped with a switching criterion allowing to properly address in a stable way poor excitation scenarios. The estimator's stability properties are analyzed by Input-to-State Stability arguments. The practical characteristics of the proposed estimation approach are evaluated and compared with other existing tools by extensive simulation trials. Real experimental results are provided as well.
\end{abstract}

\section{INTRODUCTION}

The problem of estimating the amplitude, frequency and phase (AFP problem) of a single sinusoidal signal from measurements corrupted by external disturbances appears in a variety of engineering applications including active noise cancellation, vibrations suppression (see [1] and the references therein) and periodic disturbance rejection (see [2], [3], [4], [5]), to mention a few. Recent research activities focused on the robust sinusoid estimation problem in presence of both structured and unstructured uncertainties (see, for example, [6], [7], [8], [9], [10], [11] and [12] and the references cited therein). Indeed, in the recent paper [13] the authors propose a pre-filtering scheme that allows at the same time to deal with structured disturbances and to generate additional variables that are useful to generate a robust estimation of the sinusoid parameters.

The ability of dealing with structured and unstructured uncertainties has important practical implications in applications scenarios in which bias and drift phenomena occur frequently.

B. Chen is with the Dept. of Electrical and Electronic Engineering at the Imperial College London, UK (boli.chen10@imperial.ac.uk) .

G. Pin is with Electrolux Professional S.p.A, Italy, e-mail: (gilbertopindalice.it).

W. M. $\mathrm{Ng}$ and C.K. Lee are with the Dept. of Electrical and Electronic Engineering, The University of Hong Kong, Hong Kong (wmng@eee.hku.hk, ckleedeee.hku.hk).

S. Y. R. Hui is with the Dept. of Electrical and Electronic Engineering, The University of Hong Kong, Hong Kong, and also with the Dept. of Electrical and Electronic Engineering at the Imperial College London, UK (ronhuideee.hku.hk; r.huidimperial.ac.uk).

T. Parisini is with the Dept. of Electrical and Electronic Engineering at the Imperial College London, UK, and also with the Dept. of Engineering and Architecture at the University of Trieste, Italy (t.parisini@gmail.com).
For example, in [9], an extension of the adaptive notch-filtering (ANF) scheme for the biased sinusoid estimation problem has been proposed in order to remove the limitation of the conventional ANF that is natively applicable for unbiased measurement. On the other hand, the class of Phase-LockedLoop (PLL) methodology is also commonly exploited for constructing nonlinear estimation methods that are capable to provide reliable estimates in a noisy environment (see [14], [15]). However, conventional PLL-based approaches suffer from a low-frequency ripple in presence of a dc bias. A modified method which consists of the enhanced phase-locked loop [14] and an extra estimator for the dc component is introduced in [16] to overcome such a drawback. In [8], a recursive method, relying on second-order generalized integrators is presented to reconstruct the unbiased sinusoid from a biased measurement. Moreover, another fourth-order frequency estimator that can cope with bias is proposed in [7]: by adopting a switching strategy, this algorithm provides extra attenuation of high-frequency noise in steady state. However, the switching algorithm has to be reset if the nominal frequency changes.

Adaptive observers represent a valid alternative to the aforementioned methods offering simultaneous estimation of states and parameters. Adaptive observer have been extensively characterized from a control-theoretical perspective, being able to achieve global or semi-global stability. In this framework, the frequency of the periodic signal is often modelled as an unknown parameter to be identified through an adaptive observer algorithm (see, for instance, [17] and [18], and the references cited therein). A notable feature of adaptive observer schemes is the possibility of carrying out multisinusoidal estimation by expanding the dynamic model with a suitable system transformation (see, for example, [19], [10], [11], and the very recent paper [20]). The flexibility of being able to address more general scenarios that include more than one sinusoid and also that allow simultaneous estimation of sinusoids parameters and state variables is an advantage of observer-based AFP schemes.

Based on some recent preliminary results presented in [21], this paper deals with a "dual-mode" estimation scheme, incorporating a switching algorithm (depending on the realtime excitation level) into an adaptive observer-based sinusoidal estimator. In comparison with the traditional adaptive estimators that rely on an integral type persistency of excitation condition, the devised method allows to check the excitation level in real-time thus avoiding the need for on-line 
approximate computation of integrals. The stability properties of the devised method are analyzed in terms of Input-toState Stability (ISS) arguments thus coping with bounded measurement noise.

The paper is organized as follows: Section II introduces several useful notations and basic definitions; the problem formulation is given in Section III. In Section IV, the robust estimation algorithm based on an adaptive observer is proposed. Then, the ISS analysis of the proposed technique is dealt with in Section V. Finally, simulation and real experimental results showing the effectiveness of the algorithm dealt with in the paper are given in Section VII.

\section{Notation AND BASIC Definitions}

Let $\mathbb{R}, \mathbb{R}_{\geq 0}$ and $\mathbb{R}_{>0}$ denote the real, the non-negative real and the strict positive real sets of numbers, respectively. Given a vector $\mathbf{x} \in \mathbb{R}^{n}$, we will denote as $|\mathbf{x}|$ the Euclidean norm of $\mathbf{x}$. Given a time-varying vector $\mathbf{x}(t) \in \mathbb{R}^{n}$, $t \in \mathbb{R}_{\geq 0}$ we will denote as $\|\mathbf{x}\|_{\infty}$ the quantity $\|\mathbf{x}\|_{\infty}=$ $\sup _{t>0}|\mathbf{x}(t)|$. Given a matrix $\mathbf{A} \in \mathbb{R}^{n \times n}$, then $\|\mathbf{A}\|$ will denote $\max _{\mathbf{x} \in \mathbb{R}^{n} \backslash 0}\{|\mathbf{A x}| /|\mathbf{x}|\}$.

The notions of functions of class $\mathcal{K}$, class $\mathcal{K}_{\infty}$, and class $\mathcal{K} \mathcal{L}$ are used to characterize stability properties. A function $\alpha: \mathbb{R}_{\geq 0} \rightarrow \mathbb{R}_{\geq 0}$ belongs to the class $\mathcal{K}$ if it is continuous, strictly increasing and $\alpha(0)=0$. If, in addition $\lim _{s \rightarrow \infty}=\infty$ then it belongs to the class $\mathcal{K}_{\infty}$. A continuous function $\beta: \mathbb{R}_{\geq 0} \times \mathbb{R}_{\geq 0} \rightarrow \mathbb{R}_{\geq 0}$ belongs to the class $\mathcal{K} \mathcal{L}$ if, for any fixed $t \in \mathbb{R}_{\geq 0}$, the function $\beta(\cdot, t)$ is a $\mathcal{K}$-function with respect to the first argument and if, for any fixed $s \in \mathbb{R}_{\geq 0}$, the function $\beta(s, t)$ is monotonically decreasing with respect to $t$ and $\lim _{t \rightarrow \infty} \beta(s, t)=0$. Given an $i$-times differentiable vector of signals $\mathbf{u}(t) \in \mathbb{R}^{n}, \forall t \in \mathbb{R}_{\geq 0}$, we denote by $\frac{d^{i}}{d t^{i}} \mathbf{u}(t)$ the vector of $i$-th derivative signals.

Consider the following dynamical system

$$
\dot{\mathbf{x}}=f(\mathbf{x}, \mathbf{u})
$$

with $\mathbf{x} \in \mathbb{R}^{n}, \mathbf{u} \in \mathbb{R}^{m}, f(0,0)=0$ and $f(\mathbf{x}, \mathbf{u})$ locally Lipschitz in $\mathbb{R}^{n} \times \mathbb{R}^{m}$.

Definition 2.1 (ISS): The system (1) is ISS (Input-to-State Stable) if there exist a $\mathcal{K} \mathcal{L}$-function $\beta(\cdot, \cdot)$ and a class $\mathcal{K}$ function such that, for any input $\mathbf{u} \in \mathbb{R}^{m}$ and any initial condition $\mathbf{x}_{0} \in \mathbb{R}^{n}$, the trajectory of the system verifies

$$
|\mathbf{x}(t)| \leq \beta\left(\left|\mathbf{x}_{0}\right|, t\right)+\gamma\left(\|\mathbf{u}\|_{\infty}\right)
$$

Definition 2.2 (ISS-Lyapunov Function): A function $V$ : $\mathbb{R}^{n} \rightarrow \mathbb{R}_{\geq 0}$ of class $\mathcal{C}^{1}$ is an ISS-Lyapunov function for (1) if there exist three $\mathcal{K}_{\infty}$-functions $\underline{\alpha}(\cdot), \bar{\alpha}(\cdot), \alpha(\cdot)$ and a $\mathcal{K}$ function $\mathcal{X}(\cdot)$ such that

$$
\underline{\alpha}(|\mathbf{x}|) \leq V(\mathbf{x}) \leq \bar{\alpha}(|\mathbf{x}|), \quad \forall \mathbf{x} \in \mathbb{R}^{n}
$$

and

$$
|\mathbf{x}| \geq \mathcal{X}(|\mathbf{u}|) \Rightarrow \frac{\partial V}{\partial \mathbf{x}} f(\mathbf{x}, u) \leq-\alpha(|\mathbf{x}|), \forall \mathbf{x} \in \mathbb{R}^{n}, \forall \mathbf{u} \in \mathbb{R}^{m}
$$

Theorem 2.1 ([22]): The system (1) is ISS if and only if it admits an ISS-Lyapunov function.

\section{Problem Statement And Pre-Filtering Design}

Consider a nominal sinusoidal signal described by

$$
\left\{\begin{array}{l}
s(t)=A \sin (\vartheta(t)) \\
\dot{\vartheta}(t)=\omega^{*} \\
\vartheta_{0}=\phi
\end{array}, \quad t \in \mathbb{R}_{\geq 0} .\right.
$$

where $A, \omega^{*}$, and $\vartheta$ denote the amplitude, angular frequency and phase, respectively.

The AFP estimation problem deals with estimating $A, \omega^{*}$, and $\vartheta$ from the following perturbed measurement:

$$
\hat{y}(t)=s(t)+\sum_{k=1}^{n_{d}} b_{k} t^{k-1}+d(t), \quad t \in \mathbb{R}_{\geq 0}
$$

where the term $\sum_{k=1}^{n_{d}} b_{k} t^{k-1}$ represents a time-polynomial structured perturbation, with $b_{k}$ unknown for any $k \in$ $\left\{1, \ldots, n_{d}\right\}$, and $n_{d}$ is chosen a-priori by the designer depending on the expected type of structured perturbations. More specifically, setting $n_{d}=1$ allows to cope with unknown bias and offsets (for example, the ones typically affecting physical transducers and A/D converters). Setting $n_{d}=2$ entails the presence of drift phenomena (for instance, the ones affecting several sensing devices that are sensitive to temperature variations) ${ }^{1}$. The term $d(t)$ characterizes a bounded unstructured measurement noise, that is, we assume

$$
|d(t)| \leq \bar{d}, \quad t \in \mathbb{R}_{\geq 0} .
$$

where $\bar{d}$ is an a-priori known positive scalar. Moreover, we also assume that a (possibly conservative) known upper bound $\bar{\omega}$ on the angular frequency $\omega^{*}$ is available, that is

$$
\omega^{*} \leq \bar{\omega} .
$$

To deal with the structured perturbation term $\sum_{k=1}^{n_{d}} b_{k} t^{k-1}$ appearing in (6), we extend the pre-filtering strategy proposed in [23], [13] (see also the alternative GPI observer approach [24]). To this end, we address the AFP estimation problem for the noise-free signal

$$
y(t)=s(t)+\sum_{k=1}^{n_{d}} b_{k} t^{k-1}, \quad t \in \mathbb{R}_{\geq 0} .
$$

In the simplified setting given by (7), the pre-filtering consists in computing $1+n_{d}$ auxiliary filtered signals $x_{1}(t), x_{2}(t), \ldots$, $x_{1+n_{d}}(t)$, obtained as follows, and that will be used later on in the adaptive observer-based estimator (see (14)):

$$
\left\{\begin{array}{l}
\dot{x}_{1}(t)=\lambda\left(\beta y(t)-x_{1}(t)\right) \\
\dot{x}_{k}(t)=\lambda\left(\beta x_{k-1}(t)-x_{k}(t)\right), \forall k \in\left\{2, \ldots, 1+n_{d}\right\}
\end{array},\right.
$$

where $x_{k}(0)=x_{k 0}, k \in\left\{1, \ldots, 1+n_{d}\right\} ; \lambda$ and $\beta$ are positive design parameters to be selected by the designer. In qualitative terms, $\lambda$ determines the cut-off frequency while $\beta \in(0,1]$ acts as a damping coefficient (see Section VII

\footnotetext{
${ }^{1}$ Note that the choice of $n_{d}$ is not unique. For instance, a bias can be removed with all $n_{d} \geq 1$. Therefore, in case of a sensing devise affected by uncertain perturbations, a proper choice of $n_{d}$ has to be carried out depending on the a-priori knowledge about the possible structured uncertainties in the specific application.
} 
for more details). Defining $\mathbf{x}(t) \triangleq\left[x_{1}(t), \ldots, x_{1+n_{d}}(t)\right]^{\top}$, a state-space realization of the filter evolving from arbitrary initial conditions $\mathbf{x}_{0} \in \mathbb{R}^{1+n_{d}}$ is

$$
\left\{\begin{array}{l}
\dot{\mathbf{x}}(t)=\mathbf{A}_{\lambda, \beta} \mathbf{x}(t)+\boldsymbol{b}_{\lambda, \beta} y(t) \\
x_{1+n_{d}}(t)=\mathbf{c}^{\top} \mathbf{x}(t)
\end{array}\right.
$$

where

$$
\mathbf{A}_{\lambda, \beta}=\left[\begin{array}{ccccc}
-\lambda & 0 & \cdots & \cdots & 0 \\
\beta \lambda & -\lambda & \ddots & & \vdots \\
0 & \ddots & \ddots & \ddots & \vdots \\
\vdots & \ddots & \ddots & \ddots & 0 \\
0 & \cdots & 0 & \beta \lambda & -\lambda
\end{array}\right], \quad \mathbf{b}_{\lambda, \beta}=\left[\begin{array}{c}
\beta \lambda \\
0 \\
\vdots \\
0
\end{array}\right],
$$

and

$$
\mathbf{c}^{\top}=\left[\begin{array}{llll}
0 & \cdots & 0 & 1
\end{array}\right] .
$$

Accordingly, we have

$$
\mathbf{c}^{\top} \mathbf{A}_{\lambda, \beta}^{k} \mathbf{b}_{\lambda, \beta}=0, \forall k \in\left\{0, \ldots, n_{d}-1\right\} .
$$

Then, the time-derivatives $\dot{x}_{1+n_{d}}(t), \quad \ddot{x}_{1+n_{d}}(t), \cdots$, $\frac{d^{1+n_{d}}}{d t^{1+n_{d}}} x_{1+n_{d}}(t)$ can be computed as follows:

$$
\begin{aligned}
& \frac{d^{k}}{d t^{k}} x_{1+n_{d}}(t)=\mathbf{c}^{\top} \mathbf{A}_{\lambda, \beta}^{k} \mathbf{x}(t), \forall k \in\left\{1, \ldots, n_{d}\right\}, \\
& \frac{d^{1+n_{d}}}{d t^{1+n_{d}}} x_{1+n_{d}}(t)=\mathbf{c}^{\top} \mathbf{A}_{\lambda, \beta}^{n_{d}}\left(\mathbf{A}_{\lambda, \beta} \mathbf{x}(t)+\mathbf{b}_{\lambda, \beta} y(t)\right) .
\end{aligned}
$$

Now, we have

$$
\mathcal{L}\left[x_{k}\right](s)=H_{k}(s) \mathcal{L}[y](s) \text { with } H_{k}(s)=\frac{\lambda^{k} \beta^{k}}{(s+\lambda)^{k}} .
$$

Then, neglecting the initial conditions of the internal filter's states, the auxiliary signal can be expressed as:

$$
\begin{aligned}
& \mathcal{L}\left[x_{1+n_{d}}\right](s)=H_{1+n_{d}}(s) A \frac{s \sin (\phi)+\omega^{*} \cos (\phi)}{s^{2}+\omega^{* 2}} \\
&+H_{1+n_{d}}(s) \sum_{k=1}^{n_{d}} b_{k}(k-1) ! \frac{1}{s^{k}}
\end{aligned}
$$

and hence

$$
\begin{aligned}
\mathcal{L}\left[\frac{d^{n_{d}} x_{1+n_{d}}}{d t^{n_{d}}}\right](s)= & H_{1+n_{d}}(s) A \frac{s \sin (\phi)+\omega^{*} \cos (\phi)}{s^{2}+\omega^{* 2}} s^{n_{d}} \\
& +H_{1+n_{d}}(s) \sum_{k=1}^{n_{d}} b_{k}(k-1) ! s^{n_{d}-k} .
\end{aligned}
$$

which infers that the time-polynominal perturbation vanishes asymptoticfor the rerarrente offeviewerbriefness, let us now introduce the following vector of auxiliary derivatives:

$$
\mathbf{z}(t)=\left[\begin{array}{l}
z_{1}(t) \\
z_{2}(t)
\end{array}\right] \triangleq\left[\begin{array}{l}
\frac{d^{n_{d}}}{d t^{n_{d}}} x_{1+n_{d}}(t) \\
\frac{d^{1+n_{d}}}{d t^{1+n_{d}}} x_{1+n_{d}}(t)
\end{array}\right] .
$$

Clearly, $\mathbf{z}(t)$ tends asymptotically to a sinusoidal regime $\overline{\mathbf{z}}(t)=\left[\bar{z}_{1}(t), \bar{z}_{2}(t)\right]^{\top}$ given by

$$
\begin{aligned}
& \bar{z}_{1}(t)=\frac{d^{n_{d}}}{d t^{n_{d}}} \bar{x}_{1+n_{d}}(t)=A_{z} \sin \left(\vartheta_{z}(t)\right), \\
& \bar{z}_{2}(t)=A_{z} \omega^{*} \cos \left(\vartheta_{z}(t)\right)
\end{aligned}
$$

where

$$
\begin{aligned}
& A_{z}=A \omega^{* n_{d}}\left|H_{1+n_{d}}\left(j \omega^{*}\right)\right| \\
& \vartheta_{z}(t)=\vartheta(t)+\angle H_{1+n_{d}}\left(j \omega^{*}\right)+\frac{\pi}{2} n_{d}
\end{aligned}
$$

In view of (9), the auxiliary derivatives $\mathbf{z}(t)$ can be written in a compact form $\mathbf{z}(t)=\boldsymbol{\Lambda}\left[y(t), \mathbf{x}(t)^{\top}\right]^{\top}$, with $\boldsymbol{\Lambda}$ given by

$$
\boldsymbol{\Lambda}=\left[\begin{array}{cc}
0 & \mathbf{c}^{\top} \mathbf{A}_{\lambda, \beta}^{n_{d}} \\
\mathbf{c}^{\top} \mathbf{A}_{\lambda, \beta}^{n_{d}} \mathbf{b}_{\lambda, \beta} & \mathbf{c}^{\top} \mathbf{A}_{\lambda, \beta}^{1+n_{d}}
\end{array}\right] .
$$

As a final remark, it is immediate to see that the sinusoidal regime $\overline{\mathbf{z}}(t)$ can be described in terms of the following simple state equations:

$$
\left\{\begin{array}{l}
\dot{\overline{\mathbf{z}}}(t)=\mathbf{A} \overline{\mathbf{z}}(t)+\Omega^{*} \mathbf{A}_{1} \overline{\mathbf{z}}(t) \\
\bar{z}_{1}(t)=\mathbf{C} \overline{\mathbf{z}}(t)
\end{array}\right.
$$

by setting the initial conditions as

$$
\overline{\mathbf{z}}(0)=\left[\begin{array}{c}
A_{z} \sin \left(\vartheta_{0}+\angle H_{1+n_{d}}\left(j \omega^{*}\right)+\frac{\pi}{2} n_{d}\right) \\
A_{z} \omega^{*} \cos \left(\vartheta_{0}+\angle H_{1+n_{d}}\left(j \omega^{*}\right)+\frac{\pi}{2} n_{d}\right)
\end{array}\right],
$$

and where $\Omega^{*}=\omega^{* 2}, \mathbf{A}=\left[\begin{array}{ll}0 & 1 \\ 0 & 0\end{array}\right], \mathbf{A}_{1}=\left[\begin{array}{ll}0 & 0 \\ -1 & 0\end{array}\right]$, and $\mathbf{C}=\left[\begin{array}{ll}1 & 0\end{array}\right]$.

\section{The ADAPTIVE OBSERVER}

Consider again the perturbed measurement signal $\hat{y}(t)$ given by (6). Let us denote by $\hat{\mathbf{x}}(t)$ the actual state vector of the pre-filter, driven by the noisy signal $\hat{y}(t)$ and evolving from an arbitrary initial condition $\hat{\mathbf{x}}_{0}$ as follows:

$$
\left\{\begin{array}{l}
\dot{\hat{\mathbf{x}}}=\mathbf{A}_{\lambda, \beta} \hat{\mathbf{x}}(\mathbf{t})+\mathbf{b}_{\lambda, \beta} \hat{\mathbf{y}}(\mathbf{t}) \\
\hat{x}_{1+n_{d}}(t)=\mathbf{c}^{\top} \hat{\mathbf{x}}(t),
\end{array}\right.
$$

Now, let $\hat{\mathbf{z}}(t)$ be the vector of the computable perturbed counterpart of $\mathbf{z}(\mathbf{t})$ :

$$
\hat{\overline{\mathbf{z}}}(t)=\boldsymbol{\Lambda}\left[\hat{y}(t), \hat{\mathbf{x}}(t)^{\top}\right]^{\top} .
$$

By introducing the estimated state $\hat{\mathbf{z}}(t)$ and the estimated squared-frequency $\hat{\Omega}(t)=(\hat{\omega}(t))^{2}$, the following adaptive observer is proposed:

$$
\left\{\begin{array}{l}
\dot{\hat{\mathbf{z}}}(t)=(\mathbf{A}-\mathbf{L} \mathbf{C}) \hat{\mathbf{z}}(t)+\mathbf{L} \mathbf{C} \hat{\overline{\mathbf{z}}}(t)+\mathbf{A}_{1} \hat{\overline{\mathbf{z}}}(t) \hat{\Omega}(t)+\xi(t) \dot{\hat{\Omega}}(t) \\
\dot{\xi}(t)=(\mathbf{A}-\mathbf{L} \mathbf{C}) \xi(t)+\mathbf{A}_{1} \hat{\overline{\mathbf{z}}}(t) \\
\dot{\hat{\Omega}}(t)=-\mu \xi(t)^{\top}(\hat{\mathbf{z}}(t)-\hat{\overline{\mathbf{z}}}(t))
\end{array}\right.
$$

where $\mu$ is an arbitrary positive constant and $\mathbf{L}$ is the observer gain, obtained by assigning the eigenvalues of the observer such that $\mathbf{A}-\mathbf{L C}$ is Hurwitz.

Given $\hat{\Omega}(t)$ and $\hat{\mathbf{z}}(t)$ and assuming, for the moment, that

$$
\hat{\Omega}(t)>0, \forall t,
$$

then, the filtered regime amplitude and phase $\left(A_{z}, \vartheta_{z}\right)$ defined 
in (10) are estimated on-line by

$$
\hat{A}_{z}(t)=\sqrt{\left(\hat{\Omega}(t) \hat{z}_{1}(t)^{2}+\hat{z}_{2}(t)^{2}\right) / \hat{\Omega}(t)},
$$

and

$$
\hat{\vartheta}_{z}(t)=\angle\left[\hat{z}_{2}(t)+j \hat{\omega}(t) \hat{z}_{1}(t)\right]
$$

Thanks to the availability of $\hat{A}_{z}, \hat{\omega}, \hat{\vartheta}_{z}$ related to the auxiliary signal $\bar{z}_{1}(t)$, from (10), with the assumption (15), we obtain the following estimates of the original parameters:

$$
\hat{A}(t)=\frac{\hat{A}_{z}(t)}{\hat{\omega}(t)^{n_{d}}}\left(\frac{1}{\left|H_{1+n_{d}}(j \hat{\omega}(t))\right|}\right),
$$

and

$$
\hat{\vartheta}(t)=\hat{\vartheta}_{z}(t)-\angle H_{1+n_{d}}(j \hat{\omega}(t))-n_{d} \frac{\pi}{2} .
$$

After some algebra, we finally get:

$$
\begin{aligned}
& \hat{A}(t)=\frac{\hat{A}_{z}(t)}{\hat{\omega}(t)^{n_{d}}}\left(\frac{\left(\lambda^{2}+\hat{\omega}(t)^{2}\right)^{\frac{1}{2}}}{\lambda \beta}\right)^{1+n_{d}}, \\
& \hat{\vartheta}(t)=\hat{\vartheta}_{z}(t)+\left(1+n_{d}\right) \arctan \left(\frac{\hat{\omega}(t)}{\lambda}\right)-n_{d} \frac{\pi}{2} .
\end{aligned}
$$

Assumption (15) is needed for (16) and (18) to be wellposed at any time-instant $t$. Let us remove the need for (15) by resorting to the following adaptive amplitude estimators. To this end, note that (16) can be interpreted as a time-varying constraint for $A_{z}(t)$, that depends on the frequency estimate $\hat{\Omega}(t)$ and on the instantaneous values of the filtered signals $\hat{z}_{1}(t)$ and $\hat{z}_{2}(t)$. Introducing the time-varying residual

$$
R\left(\hat{A}_{z}(t), t\right) \triangleq \hat{A}_{z}(t) \hat{\omega}(t)-\sqrt{\hat{\Omega}(t) \hat{z}_{1}(t)^{2}-\hat{z}_{1}(t)^{2}},
$$

the following adaptation law can be designed

$$
\begin{aligned}
& \dot{\hat{A}}_{z}(t)=-\mu_{A} \frac{\partial R\left(\hat{A}_{z}, t\right)}{\partial \hat{A}_{z}} R\left(\hat{A}_{z}, t\right) \\
& \quad=-\mu_{A} \hat{\omega}(t)\left[\hat{\omega}(t) \hat{A}_{z}(t)-\sqrt{\left(\hat{\Omega}(t) \hat{z}_{1}(t)^{2}+\hat{z}_{2}(t)^{2}\right)}\right]
\end{aligned}
$$

where $\hat{A}_{z}(0)=0$ and $\mu_{A} \in \mathbb{R}_{>0}$ is a tuning gain set by the designer to ensure the asymptotic convergence of $R\left(\hat{A}_{z}(t), t\right)$ to 0 . Hence, the estimate of the filtered amplitude $\hat{A}_{z}(t)$ can be computed through (21) without the need of assuming (15). Using $\hat{A}_{z}(t)$ provided by (21), the following adaptive algorithm can be finally used to estimate the original amplitude:

$$
\dot{\hat{A}}(t)=-\mu_{A} \hat{\omega}^{n_{d}}\left(\hat{A}(t) \hat{\omega}^{n_{d}}-\frac{\hat{A}_{z}(t)}{\left|H_{1+n_{d}}(j \hat{\omega}(t))\right|}\right),
$$

with $\hat{A}(0)=0$. In Section VII, the amplitude estimate given by (22) is evaluated in simulation.

\section{ISS PROPERTY OF THE ADAPTIVE ESTIMATOR}

Let us first analyze the stability of the filter's dynamics. Introducing the error vector with respect to $\overline{\mathbf{x}}(t)$, which is driven by a choice of the filter's initial state $\overline{\mathbf{x}}_{0}$ such that the projection on the $\mathbf{z}$ subspace of the filtered state trajectory $\overline{\mathbf{x}}(t)$ matches the stationary sinusoidal behaviour since the very beginning,

$$
\tilde{\mathbf{x}}(t) \triangleq \hat{\mathbf{x}}(t)-\overline{\mathbf{x}}(t)
$$

and noticing that $d(t)=\hat{y}(t)-y(t)$, the dynamics of $\tilde{\mathbf{x}}(t)$ can be written as:

$$
\left\{\begin{array}{l}
\dot{\tilde{\mathbf{x}}}(t)=\mathbf{A}_{\lambda, \beta} \tilde{\mathbf{x}}(t)+\mathbf{b}_{\lambda, \beta} d(t) \\
\tilde{\mathbf{x}}(0)=\hat{\mathbf{x}}_{0}-\overline{\mathbf{x}}_{0}
\end{array}\right.
$$

Being $\mathbf{A}_{\lambda, \beta}$ Hurwitz, it is well-known from standard linear systems theory that the filter is ISS with respect to the additive noise $d(t)$. In particular, it can be shown that ISS asymptotic gain of this filter is given by $\gamma_{x}(s)=\frac{a_{2}}{a_{1}} \mathcal{X}(s)$, in which $\mathcal{X}(s)=\frac{2\|\mathbf{P}\|\left|\mathbf{b}_{\lambda, \beta}\right|}{1-\epsilon} s$ with $s \in \mathbb{R}_{\geq 0}$ and $0<\epsilon<1, \mathbf{P}$ is a positive definite matrix that solves the Lyapunov equation $\mathbf{P A}_{\lambda, \beta}+\mathbf{A}_{\lambda, \beta}^{\top} \mathbf{P}=-\mathbf{I}$ and $a_{1}, a_{2}$ are two positive scalars, such that $a_{1}|\tilde{\mathbf{x}}|^{2} \leq \tilde{\mathbf{x}}^{\top} \mathbf{P} \tilde{\mathbf{x}} \leq a_{2}|\tilde{\mathbf{x}}|^{2}, \quad \forall \tilde{\mathbf{x}}$.

In view of the ISS property of the linear auxiliary filter (23), for any arbitrary $\nu \in \mathbb{R}_{>0}$ and for any finite-norm initial error $\tilde{\mathbf{x}}_{0}$, the error vector $\tilde{\mathbf{x}}(t)$ enters in a closed ball of radius $\gamma_{x}\left(\|d\|_{\infty}\right)+\nu \leq \gamma_{x}(\bar{d})+\nu$ in finite time $T_{\tilde{\mathbf{x}}_{0}, \nu}$. Thanks to (13), the vector $\tilde{\mathbf{z}}(t) \triangleq \hat{\overline{\mathbf{z}}}(t)-\overline{\mathbf{z}}(t)$ enters in finite-time $T_{\delta_{z}}=T_{\tilde{\mathbf{x}}_{0}, \nu}$ (for the sake of simplifying the notation, we have dropped the dependence of the reach-time $T_{\delta_{z}}$ on initial conditions) in a closed ball of radius $\gamma_{z}(\bar{d})+\delta_{z}$ centered at the origin, with

$$
\delta_{z}=\bar{\lambda} \nu ; \quad \gamma_{z}(s)=\bar{\lambda}\left(\gamma_{x}(s)+s\right), \forall s \in \mathbb{R}_{\geq 0},
$$

where $\bar{\lambda}=\|\boldsymbol{\Lambda}\|$. Thus, the boundedness of $\hat{\overline{\mathbf{z}}}(t)$ is justified due to the boundedness of $\overline{\mathbf{z}}(t)$ and the equality $\hat{\overline{\mathbf{z}}}(t)=\tilde{\mathbf{z}}(t)+\overline{\mathbf{z}}(t)$.

Now, we address the stability of the adaptive observer. To this end, let us introduce some instrumental error variables: $\tilde{\mathbf{z}}(t) \triangleq \hat{\mathbf{z}}(t)-\overline{\mathbf{z}}(t), \tilde{\Omega}(t) \triangleq \hat{\Omega}(t)-\Omega^{*}$, and $\psi(t) \triangleq \tilde{\mathbf{z}}(t)-$ $\xi(t) \tilde{\Omega}(t)$. Then, we have:

$$
\begin{array}{r}
\dot{\tilde{\mathbf{z}}}(t)=(\mathbf{A}-\mathbf{L} \mathbf{C}) \tilde{\mathbf{z}}(t)+\left(\mathbf{L} \mathbf{C}+\Omega^{*} \mathbf{A}_{1}\right) \tilde{\overline{\mathbf{z}}}(t)+\tilde{\Omega}(t) \mathbf{A}_{1} \hat{\overline{\mathbf{z}}}(t) \\
+\xi(t) \dot{\hat{\Omega}}(t), \\
\dot{\tilde{\Omega}}(t)=-\mu \xi(t)^{\top} \xi(t) \tilde{\Omega}(t)+\mu \xi(t)^{\top}(\tilde{\overline{\mathbf{z}}}(t)-\psi(t)),
\end{array}
$$

and

$$
\dot{\psi}(t)=(\mathbf{A}-\mathbf{L} \mathbf{C}) \psi(t)+\left(\mathbf{L} \mathbf{C}+\Omega^{*} \mathbf{A}_{1}\right) \tilde{\overline{\mathbf{z}}}(t) .
$$

The following assumption is needed to address the convergence analysis of the estimator.

Assumption 1: The solution $\xi(t)$ of $\dot{\xi}(t)=(\mathbf{A}-\mathbf{L C}) \xi(t)+$ $\mathbf{A}_{1} \hat{\overline{\mathbf{z}}}(\mathbf{t})$ is instantaneously persistently exciting (IPE) in the sense that there exist a positive constant $\epsilon$ such that

$$
\xi(t)^{\top} \xi(t)>\epsilon, \forall t>0
$$

It is worth noting that, instead of the typical integral type of persistency of excitation condition (see, for instance, [7]) that requires on-line buffering by a moving time window, the exploitation of the above IPE condition in the switching algorithm presented in Section VI greatly enhances the on-line implementation of the adaptive estimation technique.

Now, the following basic stability result is given and proved. 
Theorem 5.1 (ISS of the adaptive observer system):

Suppose that Assumption 1 holds. Then, given the sinusoidal signal $s(t)$ generated by (5) and the perturbed measurement model (6), the adaptive observer as well as the frequency estimator given by (12), (13) and (14) are ISS with respect to any additive measurement perturbation such that $|d(t)| \leq \bar{d}$.

Proof: Consider the candidate Lyapunov function $V_{\psi}=\psi(t)^{\top} \mathbf{Q} \psi(t)$, where $\mathbf{Q}$ is a positive definite matrix solving the linear Lyapunov's equation: $\mathbf{Q}(\mathbf{A}-\mathbf{L} \mathbf{C})+(\mathbf{A}-\mathbf{L} \mathbf{C})^{\top} \mathbf{Q}=-\mathbf{I}$. In view of the dynamics of $\psi(t)$ obeying (26), the time-derivative of the Lyapunov function verifies the inequality

$$
\frac{\partial V_{\psi}}{\partial \psi} \dot{\psi}(t) \leq-|\psi(t)|^{2}+2|| \mathbf{Q} \||| \mathbf{L} \mathbf{C}+\Omega^{*} \mathbf{A}_{1}|| \tilde{\overline{\mathbf{z}}}(t)|| \psi(t) \mid .
$$

Hence, $V_{\psi}$ is an ISS-Lyapunov function for $\psi(t)$ with respect to the $\tilde{\mathbf{z}}(\mathbf{t})$. Moreover, the dynamics of $\tilde{\mathbf{z}}(\mathbf{t})$ is ISS with respect to disturbance $d(t)$, so that $\psi(t)$ is, in turn, ISS with respect to $d(t)$ such that $|d(t)| \leq \bar{d}$. Now, let $V_{\tilde{\Omega}}=\frac{1}{2} \tilde{\Omega}(t)^{2}$ be a candidate Lyapunov function of the frequency-estimation subsystem. Then, the derivative of $V_{\tilde{\Omega}}$ verifies the inequality

$$
\frac{\partial V_{\tilde{\Omega}}}{\partial \tilde{\Omega}} \dot{\tilde{\Omega}}(t) \leq-\mu|\xi(t)|^{2}|\tilde{\Omega}(t)|^{2}+\mu|\xi(t)||\tilde{\overline{\mathbf{z}}}(t)-\psi(t)||\tilde{\Omega}(t)|
$$

In view of (28), Assumption 1, and the boundedness of $|\xi(t)|$ (it is immediate to show that the dynamics of $\xi(t)$ is ISS with respect to the bounded input $\hat{\bar{z}}$ ), we have that $\tilde{\Omega}(t)$ is ISS with respect to $\psi(t)$ and $\tilde{\bar{z}}(t)$, which are all proven to be ISS with respect to $d(t)$ such that $|d(t)| \leq \bar{d}$.

Finally, the identity $\tilde{\mathbf{z}}(t)=\psi(t)+\xi(t) \tilde{\Omega}(t)$, and the boundedness of $|\xi(t)|$ together imply that also the state-estimation error $\tilde{\mathbf{z}}(t)$ is ISS with respect to $d(t)$ such that $|d(t)| \leq \bar{d}$.

Next, we are going to establish the relationship between the excitation condition and the observer poles location.

Lemma 5.1 (Observer Poles and Excitation): Assume that in the noise-free mode of behaviour (that is, $d(t)=0$ ), the poles of $\mathbf{A}-\mathbf{L C}$ are assigned to $\left(p_{1}, p_{2}\right)$, where $p_{1}, p_{2}=$ $a \pm j b$ with $a \in \mathbb{R}_{<0}, b \in \mathbb{R}$, such that

$$
a^{2}>b^{2}, a \in \mathbb{R}_{<0}, b \in \mathbb{R} .
$$

Then, the PE condition (27) is verified for any $t>0$ by any sinusoidal signal.

Proof: In stationary conditions, by defining $\mathbf{B}_{\xi}=$ $\left[\begin{array}{ll}0 & -1\end{array}\right]^{\top}$, the dynamic equation of $\xi(t)$, in absence of noise can be rewritten as

$$
\dot{\xi}(t)=(\mathbf{A}-\mathbf{L} \mathbf{C}) \xi(t)+\mathbf{B}_{\xi} \bar{z}_{1}(t) .
$$

Then, in the Laplace domain we have $\xi_{k}(s)=$ $G_{k}(s) \bar{z}_{1}(s), k \in\{1,2\}$, where $G_{k}(s)=\mathbf{e}_{i}^{\top}(s \mathbf{I}-\mathbf{A}+$ $\mathbf{L C})^{-1} \mathbf{B}_{\xi}$ and $\mathbf{e}_{i}$ denotes the $i$-th unit vector.

Now, letting $p \triangleq p_{1}+p_{2}$ and $q \triangleq p_{1} p_{2}$, by a simple algebra we obtain

$$
G_{1}(s)=-\frac{1}{s^{2}-p s+q}, \quad G_{2}(s)=-\frac{s-p}{s^{2}-p s+q},
$$

and

$$
\phi_{G_{1}}=\arctan \frac{p \omega}{q-\omega^{2}}, \quad \phi_{G_{2}}=\arctan \frac{p^{2} \omega-q \omega+\omega^{3}}{p q} .
$$

Owing to the structure of $G_{1}$ and $G_{2}$, the following inequality holds

$$
\xi^{\top}(t) \xi(t) \geq \sum_{k=1}^{2} g_{k}^{2} A_{z}^{2} \sin ^{2}\left(\vartheta_{z}+\phi_{G_{k}}\right),
$$

where $g_{k}=\left|G_{k}(j \bar{\omega})\right|$ and $\phi_{G_{k}}$ represents the phase shift of $G_{k}(s)$ at the frequency of the sinusoid.

Now, we show that $\phi_{G_{1}}(\omega) \neq \phi_{G_{2}}(\omega), \forall \omega>0$ by contradiction. Let us assume that there exists $\omega>0$, such that $\phi_{G_{1}}=\phi_{G_{2}}$. The hypothesis is verified if and only if

$$
\frac{p}{q-\omega^{2}}=\frac{p^{2}-q+\omega^{2}}{p q},
$$

which is equivalent to

$$
\omega^{4}+\left(p^{2}-2 q\right) \omega^{2}+q^{2}=0 .
$$

In view of (29), Eq. (30) does not admit positive roots in the variable $\omega$ (since $p^{2}-2 q=p_{1}^{2}+p_{2}^{2}>0$ and $q^{2}>0$ ). Therefore, we can conclude that $\phi_{G_{1}} \neq \phi_{G_{2}}, \forall \omega>0$. Finally, due to the phase separation property, the following inequality is verified for all $t>0$

$$
\xi^{\top}(t) \xi(t) \geq \sum_{k=1}^{2} g_{k}^{2} A_{z}^{2} \sin ^{2}\left(\vartheta_{z}+\phi_{G_{k}}\right)>0
$$

and there always exist a constant $\epsilon \in \mathbb{R}_{>0}$ that fulfils (27), thus ending the proof.

\section{SWITCHING MECHANISM BASED ON EXCITATION LEVEL}

It is worth noting that the excitation condition (27) might not be satisfied on certain time-instants, especially when the magnitude of the sinusoidal signal (5) is small compared to magnitude of disturbances and higher order harmonics. In order to avoid the estimate drift phenomena, the adaptation parameter $\mu$ is switched based on the following normalized excitation level

$$
\Phi(t)=\left(\xi(t)^{\top} \xi(t)+\rho\right)^{-1} \xi(t)^{\top} \xi(t) .
$$

where $\rho$ is a given positive scalar. We introduce a pre-defined excitation threshold $\delta$ so that

$$
\mu=0 \quad \text { if } \quad \Phi(t)<\delta \text { (poor excitation). }
$$

Notice that in [25] a different switching rule is given, in which an hysteretic excitation-based switching algorithm is employed to ensure a minimum finite duration between transitions.

Clearly, it is important to show that the estimation error remains bounded even during the poor excitation scenarios. This is carried out in the following

Lemma 6.1 (Boundedness in dis-excitation phase):

Assume that $\mu=0, \forall t \geq \bar{t}>0$, where $\bar{t}$ denotes the time-instant at which the adaptation is switched off. Then, the dynamics of the adaptive observer-based sinusoidal estimator given by (14) is ISS with respect to $d(t)$ such that $|d(t)| \leq \bar{d}$ 
and with respect to the value of the frequency estimation error before the adaptation is switched off (that is, $\tilde{\Omega}\left(\bar{t}^{-}\right)$).

Proof: In the suppressed identification phase, $\dot{\hat{\Omega}}(t)=$ $\dot{\tilde{\Omega}}(t)=0$, such that $\tilde{\Omega}(t)=\tilde{\Omega}\left(\bar{t}^{-}\right)$and the error dynamics $\tilde{\mathbf{z}}(t), \psi(t)$ evolve according to the following differential equations:

$$
\begin{gathered}
\dot{\tilde{\mathbf{z}}}(t)=(\mathbf{A}-\mathbf{L} \mathbf{C}) \tilde{\mathbf{z}}(t)+\left(\mathbf{L} \mathbf{C}+\Omega^{*} \mathbf{A}_{1}\right) \tilde{\mathbf{z}}(t)+\tilde{\Omega}(t) \mathbf{A}_{1} \hat{\overline{\mathbf{z}}}(t), \\
\dot{\psi}(t)=(\mathbf{A}-\mathbf{L C}) \psi(t)+\left(\mathbf{L C}+\Omega^{*} \mathbf{A}_{1}\right) \tilde{\overline{\mathbf{z}}}(t) .
\end{gathered}
$$

Note that the ISS properties of $\xi(t)$ and $\psi(t)$ are preserved in this scenario. Due to the identity $\tilde{\mathbf{z}}(t)=\psi(t)+\xi(t) \tilde{\Omega}(t)$, we conclude that the dynamics of $\tilde{\mathbf{z}}(t)$ admits a bound that depends on the noise level $\bar{d}$ and on the initial parametric error $\tilde{\Omega}\left(\bar{t}^{-}\right)$.

Remark 6.1 (Robustness during dynamic switching): It is worth noting that the behaviour of the estimator under alternate switches can be characterized by linking the Lyapunov bounds in any two consecutive excitation and dis-excitation infervals. Then, ip th thessibepndetomment of reviewer u the active identification phases that guarantees the asymptotic ISS property of the discrete dynamics induced by the sampling the estimator in correspondence to the transitions (see [25]).

\section{SIMULATION AND EXPERIMENTAL RESULTS}

\section{A. Simulation Results}

In this subsection, some numerical examples are given to illustrate the effectiveness of the proposed AFP methodology. The Forward-Euler discretization method with sampling period $T_{s}=1 \mathrm{~ms}$ is used in all simulations.

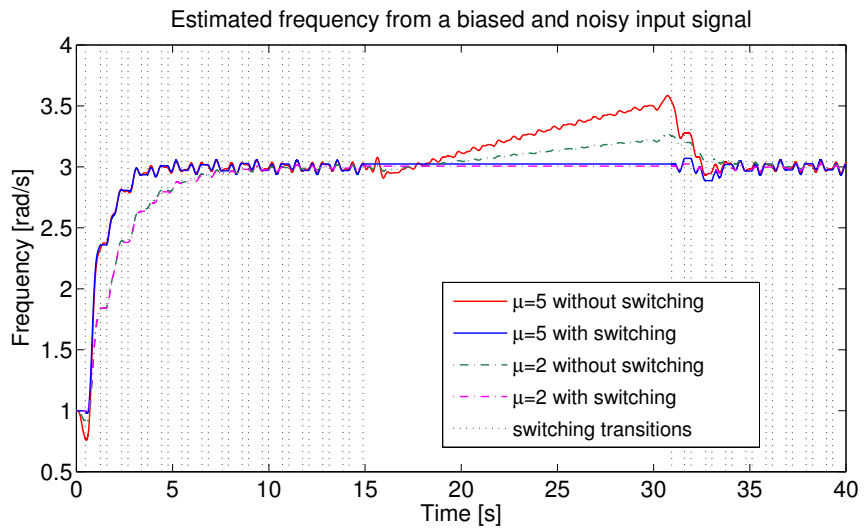

Fig. 1. Estimated frequency obtained by using the proposed AFP method with and without switching. The switching time-instants are shown by vertical dotted lines.

Example 1: Consider a biased sinusoidal measurement affected by a high-order harmonic and random noise:

$$
\hat{y}(t)=1+A(t) \sin (3 t)+\alpha(t) \sin 12 t+d(t),
$$

where $A(t)$ and $\alpha(t)$ are step-wise changing amplitudes of the sinusoid and harmonic respectively: $A(t)=5, \alpha(t)=0.5$ for $t \in[0,15), A(t)=0.5, \alpha(t)=2$ for $t \in[15,30), A(t)=$ $5, \alpha(t)=0.5$ for $t \geq 30$, while $d(t)$ is a unstructured noise with uniform distribution in the interval $[-0.5,0.5]$. With the tuning parameters set: $\lambda=2, \beta=0.7, p_{1}=-4, p_{2}=-6$, the results for the algorithm without switching for $\mu=5$ and $\mu=2$ are depicted in Fig.1 (red and green dotted lines respectively). For comparison, the estimates obtained in the same situations with the switching rule characterized by $\rho=1$ and $\delta=0.1$, is plotted in the Fig.1 as well (blue and black dotted lines respectively). In the figure, the switching timeinstants are enhanced by vertical dotted lines.

As expected, during the time-windows in which enough excitation is present, the proposed AFP estimator is able to provide reliable estimates. On the other hand, during poorlyexcited scenarios, the estimated frequency is frozen at the value taken immediately before turning-off the adaptation whereas, in case of no switching, the estimate shows a drift, as expected. Moreover, it is also worth noting that larger values of $\mu$ improve the convergence rate at the expense of weaker noise attenuation (typical trade-off between asymptotic accuracy and convergence speed). Finally, we observe that the harmonic disturbance does not influence significantly the estimation performance due to its relatively small amplitude. Larger amplitudes clearly would have generated an attractive different equilibrium regime for the estimator.

Let us now address two important aspects, namely, guidelines for the tuning of the parameters $\lambda$ and $\beta$ and the effects on the estimates of the discretization.

1) Tuning rules of $\lambda$ and $\beta$ : Consider a signal $\hat{y}(t)=$ $b_{1}(t)+3 \sin 5 t+d(t)$, where $b_{1}(t)=2$ for $t \in[0,10)$, $b_{1}(t)=1$ for $t>10$, while $d(t)$ is the measurement noise with uniform distribution in the interval $[-2.5,2.5]$. First, we apply the nominal estimator with a constant $\mu=5$ (no adaptation), and after we tune $\lambda$ and $\beta$ with the constraint of keeping constant products $\lambda \beta=4$ and $\lambda \beta=4$, respectively. The results that have been obtained are shown in Fig. 2. For the sake of comparison, the results obtained with the same election of parameters but without noise are plotted as well.

As can be noticed from the plots given in Fig. 2, the noise attenuation capabilities depend on the product of $\lambda$ and $\beta$ : smaller values of this product give rise to better noise attenuation but worse convergence speed and vice versa. Moreover, for a given value of the product of $\lambda$ and $\beta$, a reduction of $\beta$ typically yields better transient performance.

2) Estimation bias due to discretization: In the practical digital implementation of the proposed continuous-time AFP methodology a bias on the frequency estimate has to be expected due to the discretization. In order to evaluate this bias, let us assume that the filter equations (8) are discretized by a Euler method with sampling-time $T_{s}$. We immediately get

$$
X_{1+n_{d}}(z)=\frac{\left(\lambda \beta T_{s}\right)^{1+n_{d}}}{\left(z-1+\lambda T_{s}\right)^{1+n_{d}}} Y(z),
$$

where $Y(z)$ represents the discretized measurement. After discretization and some simple algebra, the $\mathcal{Z}$-transforms of the auxiliary derivatives $z_{1}, z_{2}$ are given by

$$
Z_{1}=(\lambda \beta)^{1+n_{d}} T_{s} \frac{(z-1)^{n_{d}}}{\left(z-1+\lambda T_{s}\right)^{1+n_{d}}} Y(z)
$$



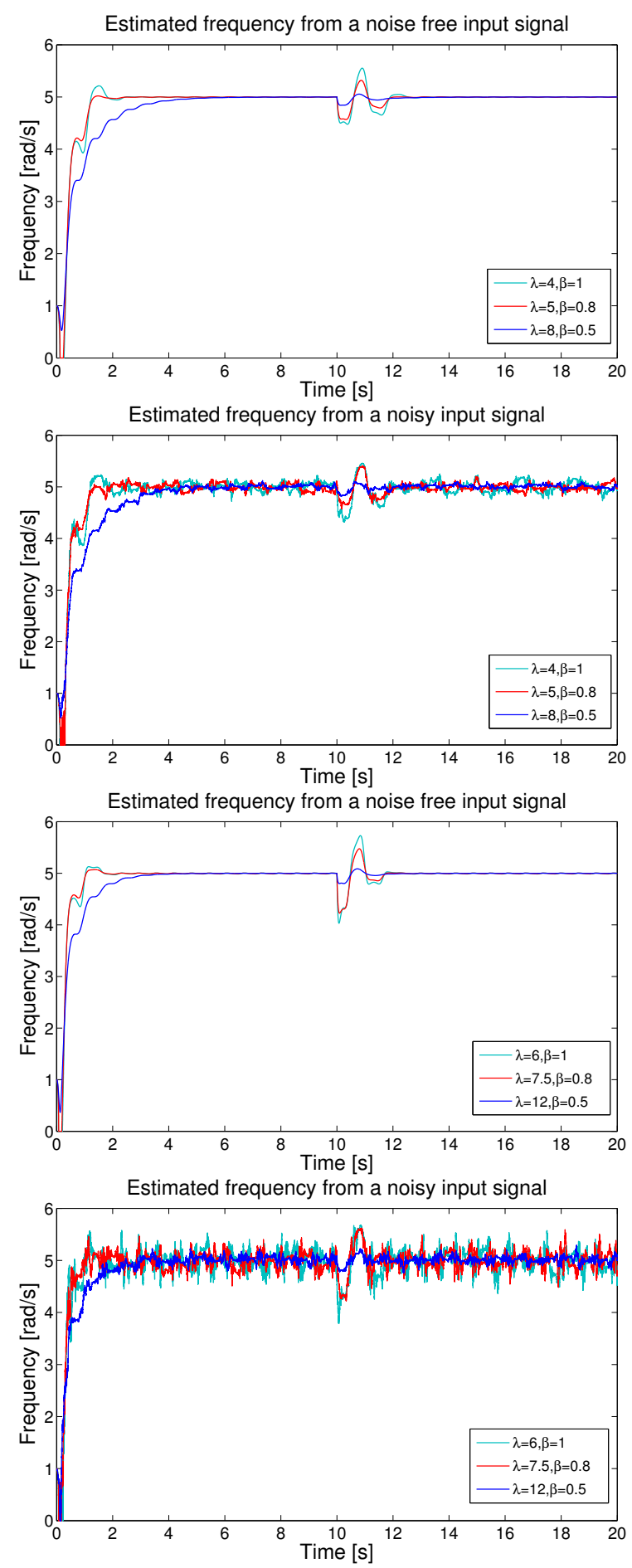

Fig. 2. Frequency tracking behavior based on the three sets of $\lambda$ and $\beta$ for a biased sinusoidal signal (top row: $\lambda \beta=4$, bottom row: $\lambda \beta=6$ )

and

$$
Z_{2}=(\lambda \beta)^{1+n_{d}} \frac{(z-1)^{1+n_{d}}}{\left(z-1+\lambda T_{s}\right)^{1+n_{d}}} Y(z) .
$$

Owing to the asymptotic sinusoidal steady-state behaviour, the squared frequency after discretization is computed by

$$
\Omega_{\text {discr }} \triangleq-\frac{z-1}{T_{s}} \frac{Z_{2}}{Z_{1}} \text {. }
$$

After some simple algebra, we get $\Omega_{\mathrm{discr}}=-\frac{(z-1)^{2}}{T_{s}^{2}}$ and then, for a given measurement with true frequency $\omega^{*}$, we have

$$
\Omega_{\mathrm{discr}}=-\frac{\operatorname{Re}\left[\left(e^{j \omega^{*} T_{s}}\right)^{2}\right]}{T_{s}^{2}}=\frac{2 \cos \left(\omega^{*} T_{s}\right)\left(1-\cos \left(\omega^{*} T_{s}\right)\right)}{T_{s}^{2}} .
$$

Thus, the steady-state value of the frequency after discretization is

$$
\omega_{\text {discr }}=\sqrt{2 \cos \left(\omega^{*} T_{s}\right)\left(1-\cos \left(\omega^{*} T_{s}\right)\right)} / T_{s} .
$$

The relationship (31) may turn out to be useful in practice to correct the effects of discretization after convergence of the estimator. To get some more insight on this important aspect, consider a noise-free signal $y(t)=2+3 \sin \omega t$ and consider increasing values of $\omega$ for a given value of the sampling-time $T_{s}=10 \mathrm{~ms}$. In Fig. 3 the corrected values generated by (31) are compared with the true values.

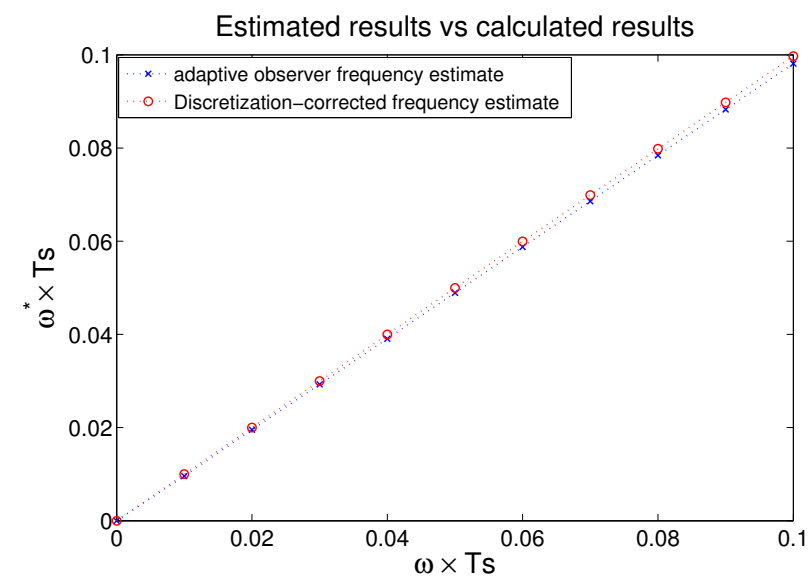

Fig. 3. Plot of $\omega T_{s}$ against $\omega^{*} T_{s}$

Example 2: In this example, a biased signal with two frequency steps is employed to compare the proposed AFP technique with two techniques available from the recent literature: the AFP method presented in [8] and the PLL-based technique in [16]. Let us assume that the signal that is perturbed by the same noise $d(t)$ as the one considered in the previous example:

$$
\hat{y}(t)=\sigma(t)+3 \sin (\omega(t) t)+d(t),
$$

with

$$
\omega(t)=\left\{\begin{array}{l}
4,0 \leq t<10 \\
6,10 \leq t<35 \\
235 \leq t<50
\end{array} \quad, \quad \sigma(t)=\left\{\begin{array}{l}
1,0 \leq t<20 \\
320 \leq t<50
\end{array}\right.\right.
$$

All the methods are initialized with the same initial condition $\hat{\omega}(0)=1$. Method [16] is tuned with: $\mu_{0}=1, \mu_{1}=$ $1, \mu_{2}=3, \mu_{3}=0.8$, while method [8] is tuned with: $K_{s}=1, \lambda=1, \omega_{s}=4, Q_{0}=(1 / \lambda) \mathbf{I}$. The adaptive parameters of the proposed method are chosen as: $\lambda=5, \beta=$ $0.7, \mu=4, p_{1}=-4, p_{2}=-6$. The simulation results are shown in Fig.4.

As can be noticed, all methods are capable to track the step-wise changing frequency with the similar response time to the first frequency value, however the PLL method [16] suffers from relatively larger overshoots for new values of the 


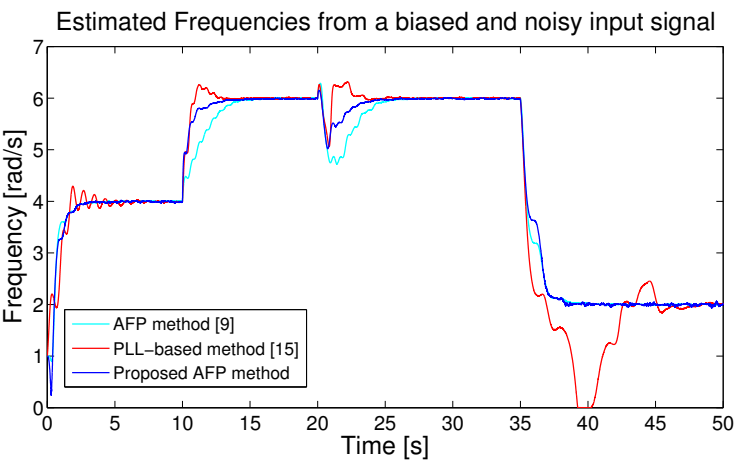

Fig. 4. Estimated Frequencies from a biased and noisy input signal

frequency and requires quite a long response time to deal with the considerable frequency drop. The AFP method [8] shows the best robustness against the noise at the cost of slowly tracking the intermediate frequency. Meanwhile, [8] is more sensitive to a bias variation. The proposed method shows the best transient performance and satisfactory capability of noise attenuation. In addition, it is worth noting that the PLL method is likely to be more sensitive to the adjustments of the tuning parameters than the other two methods.

For the sake of completeness, the behaviour of the amplitude adaptation scheme (21) is compared in Fig.5 with the outcome of the direct equation (16). The adaptive mechanism, besides resolving the division by 0 issue of (16), significantly improves the estimate in correspondence of the jumps in the frequency estimates.

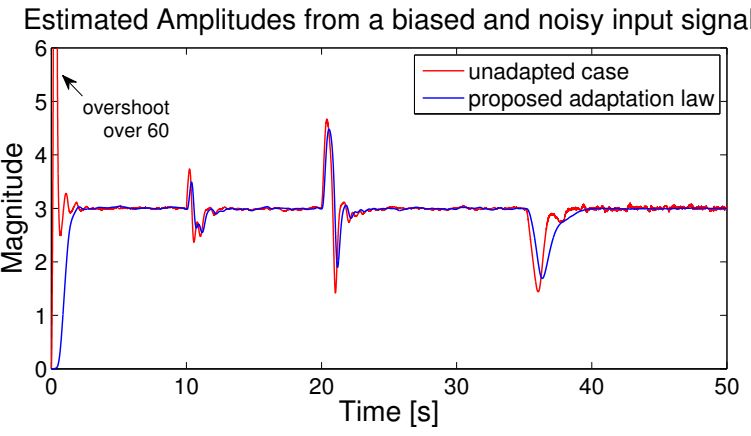

Fig. 5. Comparison of the behaviors in terms of amplitude estimation with adaptive mechanism (blue line) and unadapted algorithm(red line).

\section{B. Experimental Results}

Now, a practical implementation depicted in Fig.6 is conducted in order to evaluate the response time and accuracy of the proposed approach. Fig.7 shows a picture of the actual setup based on the Lab-Volt wind power training system, wherein the prime mover drives the wind turbine generator with a transmission belt, thereby producing an ac voltage across the generator windings. It is worth noting that the rotational speed of the prime mover to maintain the generator output $\mathrm{AC}$ frequency of $50 \mathrm{~Hz}$ is $665 \mathrm{rpm}$. During the experiment, the speed of the prime mover is programmed to emulate the intermittent nature of wind power. As a result,

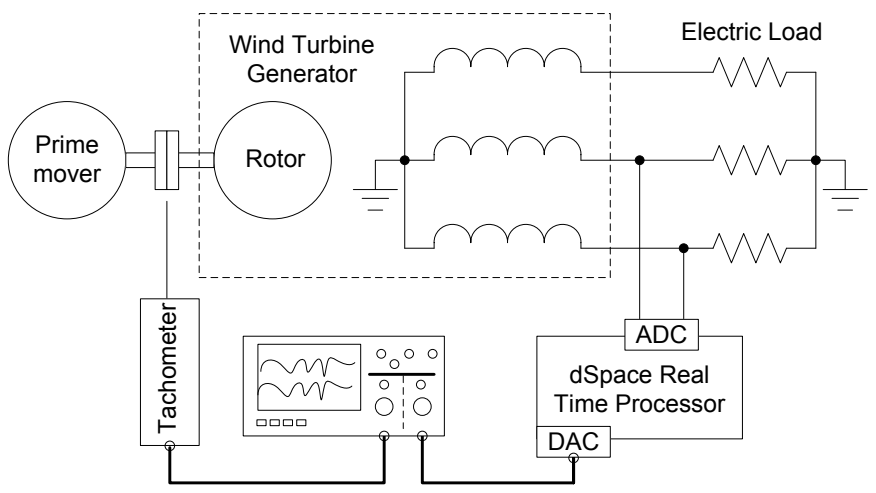

Fig. 6. Experimental setup.

the generator output voltage and frequency are not constant. Moreover, the resistive load is applied as the electrical load. The instantaneous line voltage across the generator windings is measured by an analog-to-digital-converter (ADC) with a sampling frequency of $60 \mathrm{kHz}$, while a digital-to-analog-converter (DAC) is utilized to generate the estimated frequency. Such estimates are iteratively produced by the proposed estimator, which is integrated in a digital real time processor. Finally, the measured prime mover rotational speed and the estimated frequency are captured by an oscilloscope and recorded by a high precision digital-multimeter (DMM) with 5 digits resolution.

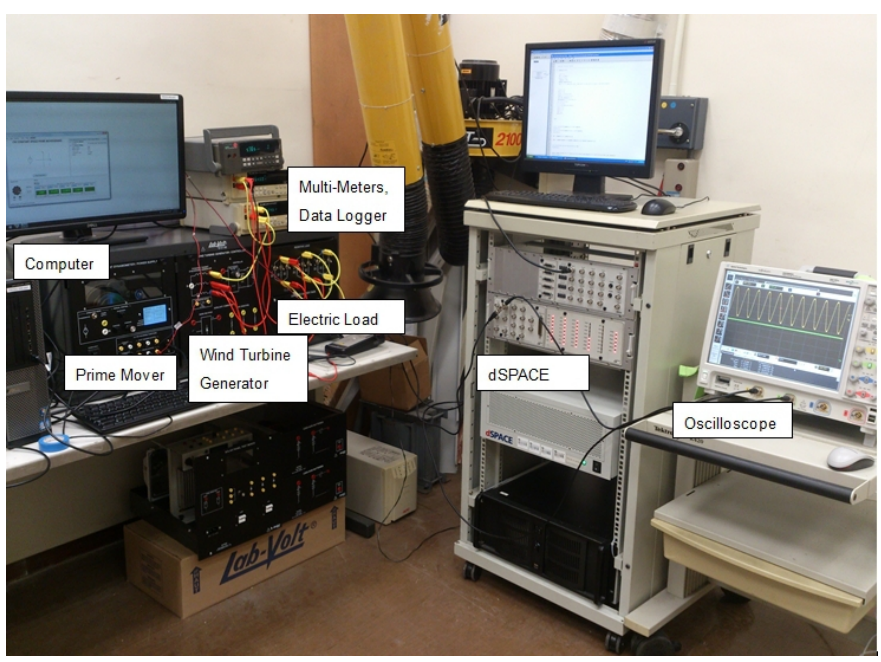

Fig. 7. Picture of the experimental setup based on Lab-Volt Wind power training system.

The dynamic behaviour of the proposed AFP algorithm is shown in Fig.8, where we observe that the estimated frequency tracks the fluctuating rotational speed of the wind turbine from $8 \mathrm{~Hz}$ to $55 \mathrm{~Hz}$ closely with almost identical profile. Fig. 9 shows the values of the identified frequency and prime mover rotational speed. The subtle differences between the frequency estimates and reading from tachometer (less than $0.25 \mathrm{mV}$ ) are due to the instrument tolerance and noise generated by the prime mover drive. Therefore, these results show that the accuracy of the proposed algorithm is limited by the resolution of the measurement equipment. It is important to note that the 
proposed AFP algorithm achieves high precision frequency estimation with an accuracy of $0.05 \mathrm{~Hz}$ resolution in this setup.

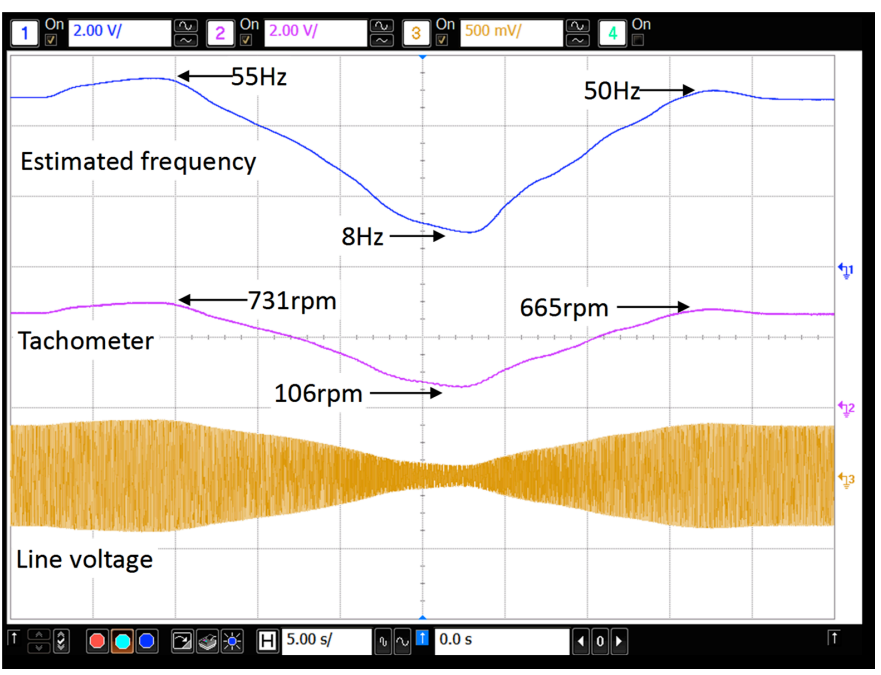

Fig. 8. Experimental results. Ch1. (bule) Estimated frequency obtained by using the proposed AFP method (20Hz/Div), Ch2. (pink) Output voltage from the analog tachometer (500rpm/Div), Ch3. (yellow) line voltage across the wind turbine generator (50V/Div). Time base: $5 \mathrm{~s} / \mathrm{Div}$.

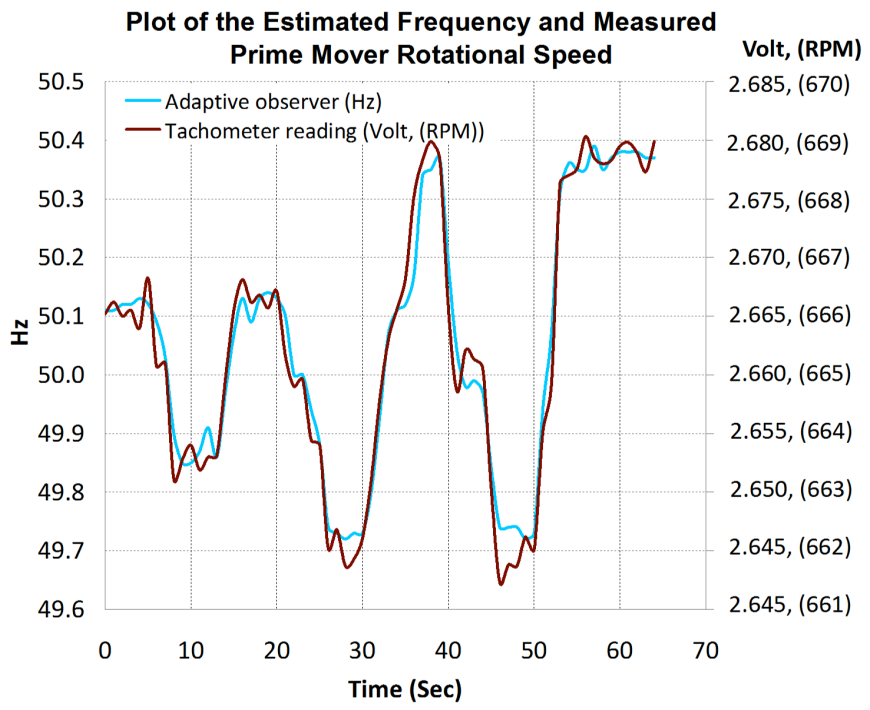

Fig. 9. Comparison of the estimated frequency obtained by using the proposed AFP method and measured prime mover rotational speed.

\section{CONCLUDING REMARKS}

In this paper, a novel dual-mode ISS-stable adaptive observer-based technique for estimation of the amplitude, frequency and phase of sinusoidal signals from measurements affected by structured and unstructured disturbances has been presented and analyzed. The estimator embeds a switching criterion that disables the adaptation in real-time under poor excitation conditions. Extensive simulations and real experiments have been carried out to show the effectiveness of the proposed adaptive algorithm.

Future research efforts will be devoted to extend the methodology to the case of multiple-frequencies estimation and to a potentially larger class of structured measurement uncertainties (some preliminary results have already been obtained in [25], [20]).

\section{REFERENCES}

[1] M. Bodson, J. S. Jensen, and S. C. Douglas, "Active noise control for periodic disturbances," IEEE Trans. on control systems technology, vol. 9, no. 1, pp. 200-205, 2001.

[2] M. Bodson and S. Douglas, "Adaptive algorithms for the rejection of sinusoidal disturbances with unknown frequency," Automatica, vol. 33, pp. 2213-2221, 1997.

[3] R. Marino, G. Santosuosso, and P.Tomei, "Robust adaptive compensation of biased sinusoidal disturbances with unknown frequency," Automatica, vol. 39, pp. 1755-1761, 2003.

[4] L. Brown and Q. Zhang, "Periodic disturbance cancellation with uncertain frequency," Automatica, vol. 40, pp. 631-637, 2004.

[5] G. Pin, "A direct approach for the frequency-adaptive feedforward cancellation of harmonic disturbances," IEEE Trans. on Signal Processing, vol. 58, no. 7, pp. 3513-3530, 2010.

[6] S. Aranovskiy, A. Bobtsov, A. Kremlev, N. Nikolaev, and O.Slita, "Identification of frequency of biased harmonic signal," European $J$. Control, vol. 2, pp. 129-139, 2010.

[7] A. Bobtsov, D. Efimov, A. Pyrkin, and A. Zolghadri, "Switched algorithm for frequency estimation with noise rejection," IEEE Trans. on Automatic Control, vol. 57, no. 9, pp. 2400-2404, 2012.

[8] G. Fedele and A. Ferrise, "Non adaptive second order generalized integrator for identification of a biased sinusoidal signal," IEEE Trans. Automatic Control, vol. 57, no. 7, pp. 1838-1842, 2012.

[9] G. Fedele, A. Ferrise, and P. Muraca, "An adaptive quasi-notch filter for a biased sinusoidal signal estimation," Santiago, 2011, pp. 1060-1065.

[10] M. Hou, "Parameter identification of sinusoids," IEEE Trans. on Automatic Control, vol. 57, no. 2, pp. 467-472, 2012.

[11] D. Carnevale, S. Galeani, and A.Astolfi, "Hybrid observer for multifrequency signals," in Proc.of IFAC workshop Adaptation and Learning in Control and Signal Processing (ALCOSP), Antalya. Elsevier, Ed., vol 10, 2010.

[12] A. A. Pyrkin, A. A. Bobtsov, D. Efimov, and A. Zolghadri, "Frequency estimation for periodical signal with noise in finite time," in Proc. of the IEEE Conf. on Decision and Control, Orlando, FL, 2011, pp. 36463651.

[13] G. Pin, B. Chen, T. Parisini, and M. Bodson, "Robust sinusoid identification with structured and unstructured measurement uncertainties," IEEE Trans. on Automatic Control, vol. 59, no. 6, pp. 1588-1593, 2014.

[14] M. Karimi-Ghartemani and A. K. Ziarani, "A nonlinear time-frequency analysis method," IEEE Trans. on Signal Processing, vol. 52, no. 6, pp. 1585-1595, 2004.

[15] M. Karimi-Ghartemani, H. Karimi, and M.R.Iravani, "A magnitude/phase-locked loop system based on estimation of frequency and in-phase/quadrature-phase amplitudes," IEEE Trans. on Industrial Electronics, vol. 51, no. 2, pp. 511-517, 2004.

[16] M. Karimi-Ghartemani, S. A. Khajehoddin, P. K. Jain, A. Bakhshai, and M. Mojiri, "Addressing DC component in PLL and notch filter algorithms," IEEE Trans. on Power Electronics, vol. 27, no. 1, pp. 7886, 2012.

[17] X. Xia, "Global frequency estimation using adaptive identifiers," IEEE Trans. on Automatic Control, vol. 47, no. 7, pp. 1188-1193, 2002.

[18] A. A. Bobtsov, "New approach to the problem of globally convergent frequency estimator," Int. J. of Adaptive Control and Signal Processing, no. 22 , pp. 306-317, 2008.

[19] R. Marino and P.Tomei, "Global estimation of $n$ unknown frequencies," IEEE Trans. Automatic Control, vol. 47, no. 8, pp. 1324-1328, 2002.

[20] B. Chen, G. Pin, and T. Parisini, "An adaptive observer-based estimator for multi-sinusoidal signals," in Proc. of the 2014 American Control Conference, Portland, OR, 2014, pp. 3450-3455.

[21] — "Adaptive observer-based sinusoid identification: structured and bounded unstructured measurement disturbances," in Proc. of the 2013 European Control Conference, Zurich, 2013.

[22] H. Khalil, Nonlinear Systems. Prentice Hall, 2001.

[23] G. Pin, T. Parisini, and M. Bodson, "Robust parametric identification of sinusoisal signals: an input-to-state stability approach," in Proc. of the IEEE Conf. on Decision and Control, Orlando, FL, 2011, pp. 61046109. 
[24] D. Martinez-Vazquez, A. Rodriguez-Angeles, and H. Sira-Ramirez, "Robust gpi observer under noisy measurements," in Proc. 2009 6th International Conference on Electrical Engineering, Computing Science and Automatic Control, 2009, pp. 1-5.

[25] G. Pin, B. Chen, and T. Parisini, "A nonlinear adaptive observer with excitation-based switching," in Proc. of the 2013 Conference on Decision and Control, Florence, 2013, pp. 4391-4398. 